\title{
Analysis of the explosive loading of open-ended steel pipes
}

\author{
N. Rushton ${ }^{1}$, G. Schleyer ${ }^{1} \&$ R. Cheesman ${ }^{2}$ \\ ${ }^{I}$ Department of Engineering, University of Liverpool, UK \\ ${ }^{2}$ Atomic Weapons Establishment (AWE), UK
}

\begin{abstract}
A programme of numerical, theoretical and experimental studies has been carried out at the University of Liverpool on the explosive loading of seamless steel pipes. Ten pipes of dimensions $800 \mathrm{~mm}$ long, $324 \mathrm{~mm}$ outside diameter and 9.5 $\mathrm{mm}$ thick were subjected to explosive loading along their mid-length using cylindrical PE4 charges detonated simultaneously from both circular faces. A range of charge sizes were used to determine the effect of the blast waves on the maximum plastic hoop strain in the pipe walls with the aim of determining the magnitude of the impulse required to initiate failure in the pipe wall. Theoretical analyses of the deformation process were investigated using equations available in the past literature as well as an attempted derivation of equations of motion used to analyse the transient deformation of the pipe where wall thinning is considered based on a constant volume assumption. A comparison of these analyses is made in this paper together with a numerical analysis of the problem using AUTODYN 2D where a von Mises material model was used to simulate the assumption of a perfectly plastic material.

Further finite element simulations were conducted to model the blast process and structural response of the experimental pipes using cylindrical charges. It was found that a Johnson-Cook strength model for the simulated pipe material in the numerical solution gives good agreement with the test data as this model attempts to account for the strain rate and strain hardening effects of the steel. The objective of the study, which is sponsored by AWE plc, Aldermaston, is to ultimately determine the failure mechanism of such a pipe under very high rates of loading.
\end{abstract}

Keywords: blast loading, seamless steel pipes, numerical simulations. 


\section{Introduction}

The detonation of an explosive releases a sudden burst of energy, which results in the propagation of a shock wave through the explosive material. The duration of these shock waves are of the order of microseconds and are characterised by a peak overpressure, $\mathrm{P}_{0}$, followed by an almost exponential decay in pressure as the shock front passes. If the detonation occurs in a confined space the typical pressure-time history for a high explosive, such as PE4, is illustrated in Fig. 1 where the pressure wave is reflected off the confining structure to produce successive peaks of decreasing magnitude.

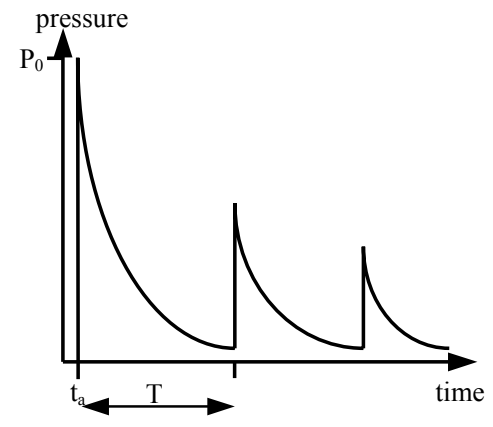

Figure 1: A typical high explosive pressure-time history for a confined detonation.
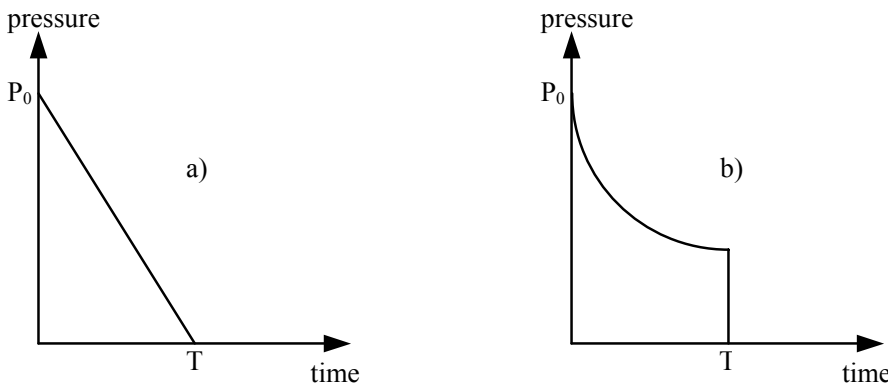

Figure 2: Pressure-time history idealisations: a) triangular decay; b) exponential decay.

Analytical methods for determining the response of structures to blast loading are usually concerned with the initial pulse duration, $T$, as this is of a far higher impulse magnitude than the successive pulses. Typical idealisations of this pulse are the triangular decay, exponential decay shown in Fig. 2.

When a containing structure, such as a cylinder, is subjected to the explosive loading by a detonated charge it can undergo substantial plastic deformation up to a maximum strain upon which subsequent motion is elastic involving both radial oscillations and other vibrations. Whereas the loading impulse duration 
lasts for microseconds, the wall response takes milliseconds to occur. This large difference between explosion loading time and vessel response time defines the loading imparted on the vessel wall as being impulsive.

\section{Theoretical analyses}

The response of cylinders to blast loading has been studied by a few authors and equations have been produced to predict the final hoop strains or radial displacements of the vessel walls. Duffey and Mitchell [1] based their analysis on a rigid-perfectly plastic material loaded by a centrally placed spherical charge. Assuming the material is not sensitive to strain hardening or rate effects and the pressure pulse is idealised as triangular, the equation predicting the final hoop strain $\left(\varepsilon_{\theta(\max )}\right)$ was found to be

$$
\varepsilon_{\theta(\max )}=\frac{\mathrm{I}^{2}}{2 \rho \sigma_{\mathrm{y}} \mathrm{h}^{2}},
$$

where I is the impulse, $\rho$ is the steel density, $\sigma_{\mathrm{y}}$ is the yield stress and $\mathrm{h}$ is the wall thickness.

A simplified analysis for an impulsively loaded cylindrical shell was produced by Fanous and Lowell [2] in which a single-degree-of-freedom (SDOF) analysis was combined with energy methods to predict the final wall displacements. The material was assumed to be ductile with an elastic-perfectly plastic material model. Other assumptions included a localised impulsive loading and assumed displacement profile to produce the following expression for the final maximum hoop strain

$$
\varepsilon_{\theta(\max )}=\frac{3 \mathrm{i}^{2}}{8 \mathrm{Mh} \sigma_{\mathrm{y}}}+\frac{\varepsilon_{\mathrm{y}}}{2},
$$

where $\mathrm{i}$ is the specific impulse, $\varepsilon_{\mathrm{y}}$ is the yield strain and $\mathrm{M}$ is the mass per unit area of the pipe.

A more recent paper was produced by Clayton [3] on the design of vessels for explosion containment. A SDOF elastic analysis was used to determine dynamic magnification factors for the response of cylindrical or spherical vessels depending on the natural frequency of the structure. Although this paper is focussed on the design of structures to resist explosions without yielding it has been shown that rule-of-thumb analyses of the stresses can be used to determine the maximum plastic deformations for impulsively loaded cylinders using the equation

$$
\varepsilon_{\mathrm{p}}=\frac{\sigma_{\mathrm{e}} \varepsilon_{\mathrm{e}}}{\sigma_{\mathrm{p}}} .
$$

where $\varepsilon_{\mathrm{p}}$ is the plastic strain, $\sigma_{\mathrm{e}}$ is the elastic stress, $\varepsilon_{\mathrm{e}}$ is the elastic strain and $\sigma_{\mathrm{p}}$ is the plastic stress.

Since none of the equations mentioned in this section consider the effects of wall thinning on the transient deformation of the shell, equations of motion have 
been derived based on a similar analysis done by Baker [4] for spherical shells. This analysis uses an incremental approach to the SDOF problem by analysing the wall displacements at different times during the deformation process. Starting from first principles, an element of the cylinder wall is analysed to determine the equations of motion as seen in Fig. 3, where $\sigma_{\theta}$ is the hoop stress, $\sigma_{\mathrm{L}}$ is the longitudinal stress, $R_{i}$ is the inner radius of the shell and $u_{r}$ is the radial displacement at any time, $\mathrm{t}$.

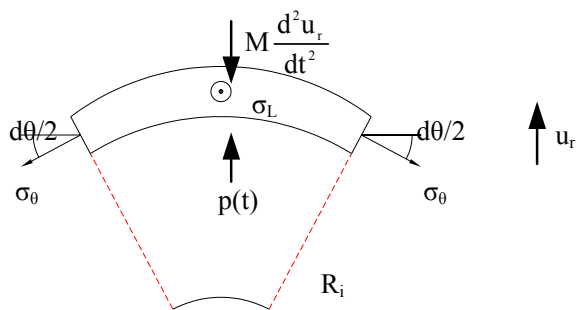

$\mathrm{d} \theta$

Figure 3: Element of a cylindrical shell under transient loading.

During the initial phase of motion, where the wall displacements are elastic and a triangular pressure pulse is assumed, the equation of motion for the cylinder wall is

$$
\frac{d^{2} u_{r}}{d t^{2}}+\omega^{2} u_{r}=\frac{p(t)}{\rho h},
$$

where the circular natural frequency, $\omega$, of the cylinder is given as

$$
\omega=\sqrt{\frac{E}{\rho\left(1-v^{2}\right) R_{i}{ }^{2}}},
$$

where E is Young's modulus and $v$ is Poisson's ratio. Solving eqn (5) and applying suitable boundary conditions gives the transient radial displacement as

$$
u_{r}=\frac{P_{0}}{\omega^{2} \rho h}\left(\frac{\sin \omega t}{\omega T}-\cos \omega t+1-\frac{t}{T}\right) .
$$

If it is assumed that the material behaves with an elastic-perfect plastic model, after the material has yielded the equation of motion becomes

$$
\frac{\mathrm{d}^{2} \mathrm{u}_{\mathrm{r}}}{\mathrm{dt}^{2}}+\frac{\sigma_{\mathrm{y}}}{\rho \mathrm{R}_{\mathrm{i}}}=\frac{\mathrm{p}(\mathrm{t})}{\rho \mathrm{h}} \text {. }
$$

This equation is solved to give the following expression for the plastic pipe displacement 


$$
\begin{gathered}
\mathrm{u}_{\mathrm{r}}=\mathrm{u}_{\mathrm{ry}}+\dot{\mathrm{u}}_{\mathrm{ry}}\left(\mathrm{t}-\mathrm{t}_{\mathrm{y}}\right)+\frac{\mathrm{P}_{0}}{\rho \mathrm{h}}\left(\frac{\mathrm{t}^{2}}{2}+\frac{\mathrm{t}_{\mathrm{y}}{ }^{2}}{2}-\mathrm{t}_{\mathrm{y}} \mathrm{t}\right)+\frac{\mathrm{P}_{0}}{\rho \mathrm{hT}}\left(\frac{\mathrm{t}_{\mathrm{y}}{ }^{2} \mathrm{t}}{2}+\frac{\mathrm{t}^{3}}{6}-\frac{\mathrm{t}_{\mathrm{y}}{ }^{3}}{3}\right), \\
+\frac{\sigma_{\mathrm{y}}}{\rho \mathrm{R}_{\mathrm{i}}}\left(\mathrm{t}_{\mathrm{y}} \mathrm{t}-\frac{\mathrm{t}_{\mathrm{y}}{ }^{2}}{2}-\frac{\mathrm{t}^{2}}{2}\right) \quad 0 \leq \mathrm{t} \leq \mathrm{t}_{\mathrm{y}}
\end{gathered}
$$

Eqn (8) applies until the pulse duration has ended after which the equation of motion becomes

$$
\mathrm{u}_{\mathrm{r}}=\mathrm{u}_{\mathrm{rT}}+\dot{\mathrm{u}}_{\mathrm{rT}}(\mathrm{t}-\mathrm{T})+\frac{\sigma_{\mathrm{y}}}{\rho \mathrm{R}_{\mathrm{i}}}\left(\mathrm{Tt}-\frac{\mathrm{t}^{2}}{2}-\frac{\mathrm{T}^{2}}{2}\right) \mathrm{t} \geq \mathrm{T} .
$$

The final wall displacement is found when the shell velocity reduces to zero. Wall thinning is accounted for at each time interval using a constant volume assumption where the incremental thickness, $\mathrm{h}_{2}$, and radius, $\mathrm{R}_{\mathrm{i} 2}$, are related to the initial values by the following quadratic

$$
\mathrm{h}_{2}=\frac{-2 \mathrm{R}_{\mathrm{i} 2}+\sqrt{4 \mathrm{R}_{\mathrm{i} 2}{ }^{2}+8 \mathrm{R}_{\mathrm{i}} \mathrm{h}+4 \mathrm{~h}^{2}}}{2} .
$$

\section{Experimental investigation}

\subsection{Material characterisation}

Two $6 \mathrm{~m}$ lengths of API 5LX-42 seamless mild steel pipes were sectioned to form cylinders of lengths $0.2 \mathrm{~m}$ and $0.8 \mathrm{~m}$ for material characterisation tests and blast tests respectively. To date only static tension tests have been performed on the pipe material. Due to the lack of sufficient material through the pipe wall thickness, specimens could not be manufactured to measure stresses and strains in the hoop direction so only longitudinal values could were obtained. The test results for the material in tension are presented in Table 1 as a range of values.

Table 1: $\quad$ Static tensile material properties for the API 5LX-42 pipe material.

$\begin{array}{lrc}\text { Yield Stress } & 289.4-344.2 & \mathrm{MPa} \\ \text { UTS } & 518.7-511.7 & \mathrm{MPa} \\ \text { Young's Modulus } & 192.2-203.1 & \mathrm{GPa} \\ \text { Poisson's Ratio } & 0.28-0.29 & \end{array}$

\subsection{Blast tests}

A series of tests were conducted on the $0.8 \mathrm{~m}$ long, $324 \mathrm{~mm}$ diameter, $9.5 \mathrm{~mm}$ thick open-ended steel pipes using centrally loaded, cylindrical PE4 charges of different masses as shown in Fig. 4. The charges were detonated simultaneously from both circular faces causing the propagation and collision of the shock waves at the centre of the explosive to impart a radial loading on the pipe mid- 
length. In order to prevent any constraint to the deformation of the wall, the pipes were supported horizontally using a combination of a wooden trestle and slings. Aligning the pipe to the horizontal served to minimise the effects of reflected pressure waves from nearby structures.

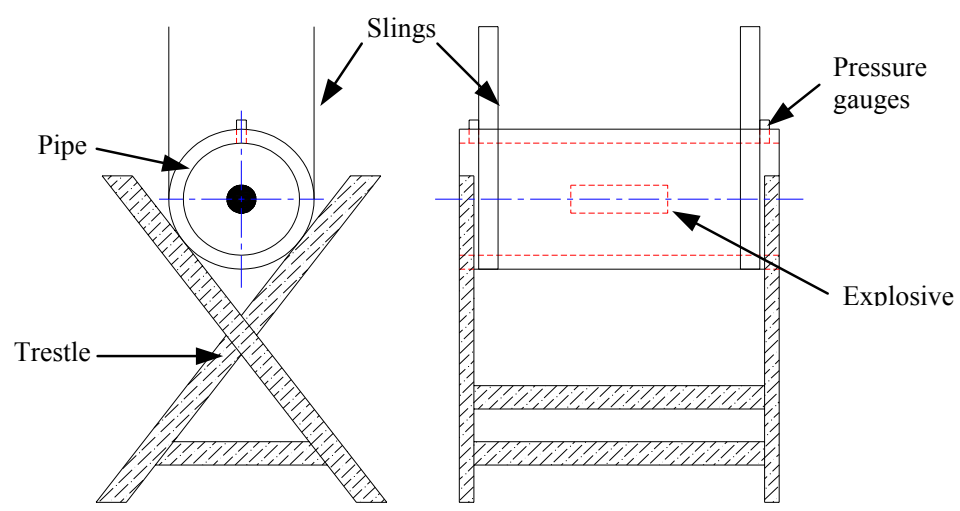

Figure 4: $\quad$ Experimental setup for the blast loading of steel pipes.

Tests started with $0.6 \mathrm{~kg}$ of PE4 with the mass increasing for different pipes in order to try and attain the mass required to initiate pipe wall failure. During each test the transient pressure was recorded using a pair of Kulite pressure gauges placed $100 \mathrm{~mm}$ from the open ends of the pipes where they would not be able to influence the deformation or failure process. The maximum hoop strain was measured at the end of each test, transient strains not being able to be recorded due to the high wall accelerations causing any attached strain gauges to debond.

\section{Numerical analysis}

The blast loading of the pipes was modelled using AUTODYN 2D [5]. Due to the axially symmetric nature of the problem the $2 \mathrm{D}$ tool was used to reduce computation time for each analysis. Two strength models were used to characterise the pipe material, namely von Mises and Johnson-Cook [6] for the comparison of the numerical results with the analytical and experimental ones respectively. It has been shown that the Johnson-Cook model is well suited for taking into account the combination of thermal softening due to high strain rate loading and strain hardening in the material, the form of the equation being given as

$$
\sigma=\left[\mathrm{A}+\mathrm{B} \varepsilon^{\mathrm{n}}\right]\left[1+\mathrm{C} \ln \dot{\varepsilon}^{*}\right]\left[1-\mathrm{T}^{* \mathrm{~m}}\right],
$$

where $\mathrm{A}$ is the yield stress, $\mathrm{B}$ is the strain hardening constant, $\mathrm{n}$ is the strain hardening exponent at a strain rate of $1 \mathrm{~s}^{-1}, \mathrm{C}$ is the strain rate constant and the final bracketed term gives an expression to account for thermal softening of the material. All constants are determined experimentally from tensile test data on 
the pipe material. However, as only static tests have been conducted to date and there is no data available for class API 5LX-42 steel, values for the constants were taken from those of 4340 steel as obtained by Johnson and Cook [6].

The pipe material was modelled as a Lagrange mesh within the Euler mesh used to represent the surrounding air and PE4 explosive. The PE4 explosive was modelled using the JWL equation of state. The setup for the simulation can be seen in Fig. 5. This setup uses a spherical PE4 charge detonated from the centre for the purpose of comparing numerical and theoretical results. The PE4 charge is modelled as a cylindrical structure for modelling the experimental setup.

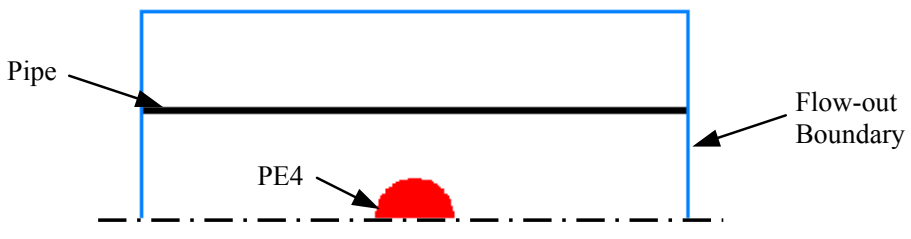

Figure 5: $\quad$ AUTODYN 2D setup of an axi-symmetric cylindrical shell loaded with a spherical PE4 charge.

\section{Results}

\subsection{Theoretical and numerical comparisons}

Approximate analyses on the deformation of open-ended steel pipes under impulsive loading were performed using the equations discussed in Section 2. In each of these analyses the explosive charge was assumed to be spherical and centrally detonated with values of the peak pressures and impulses for each charge obtained from report TM5-1300 [7] on TNT free air blast data with the relative effectiveness factor of 1.33 applied to model PE4. The other parameters used in the equations were determined from nominal pipe material properties and dimensions. The results of these theoretical analyses together with the results of numerical simulations of the problem can be seen in Fig. 6.

It can be seen in Fig. 6 that there are close approximations in hoop strains between all the analyses with the exception of that proposed by Fanous and Lowell [2] for explosive masses up to $0.6 \mathrm{~kg}$. For charge weights in excess of this value, the proposed SDOF model shows that the numerical results lie between the strains determined when wall thinning in considered and omitted, the differences between the values increasing as the mass of PE4 increases.

Out of the three theoretical analyses found in the literature, the equation proposed by Duffey and Mitchell [1] is the best approximation of the numerical solution although the difference in strain values increases with increasing charge weight. The equation proposed by Fanous and Lowell [2] consistently predicts lower strain values than the other analyses up to a charge weight approximately 1 $\mathrm{kg}$ where the Clayton [3] analysis predicts the lowest strains per mass of PE4. 
For spherical charge sizes less than $0.6 \mathrm{~kg}$ similar predictions for the final plastic hoop strain can be found using any of the theoretical analyses mentioned in Section 2 with the exception of the Fanous and Lowell [2] analysis. This analysis was not entirely suited to the selected problem as it is based on the assumption that the explosive is localised to a particular section of the cylinder wall where an assumed deformation profile is used.

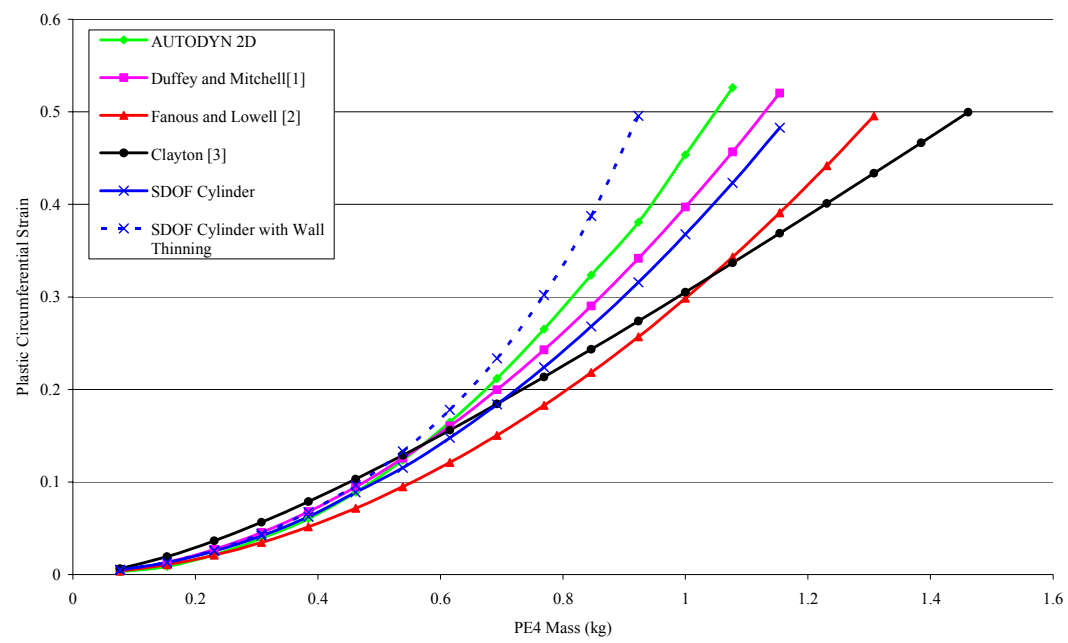

Figure 6: Comparisons between the theoretical and numerical analyses on the maximum hoop strain in a blast loaded cylindrical shell.

\subsection{Experimental and numerical comparisons}

Out of the nine blast tests conducted to date, six of the pipes underwent substantial plastic deformation and three showed signs of failure by through-wall fracture. Four results have been obtained so far from the Health and Safety Laboratories (HSL), Buxton, where the tests took place. A comparison of these results with those predicted numerically using the Johnson-Cook strength model used to simulate the pipe response is shown in Fig. 7.

The hoop strains predicted by finite element simulation are a close approximation to the experimental values for the range of charge sizes used. Differences in the results may possibly be attributed to the parameters for the Johnson-Cook material model in AUTODYN 2D being those for 4340 steel [6] and differences may occur when the material is properly characterised by high rate testing. However, from the results obtained so far, it appears that the numerical solution gives a good indication of the final plastic hoop strains in the pipe walls.

An example of the deformed pipe can be seen in Fig. 8 after explosive loading with $0.6 \mathrm{~kg}$ PE4. 


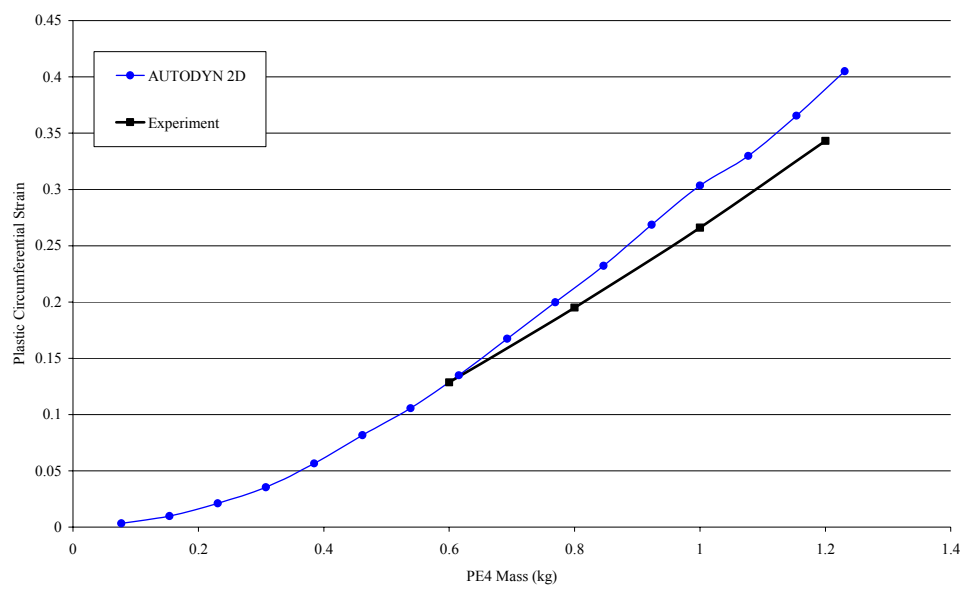

Figure 7: A comparison between the numerical predictions using the JohnsonCook material model and experimental results for the blast loaded pipes.

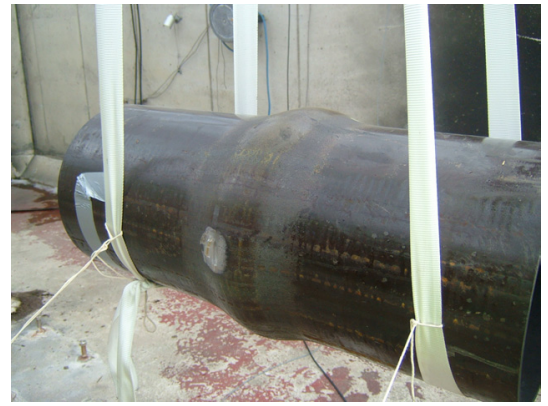

(a)

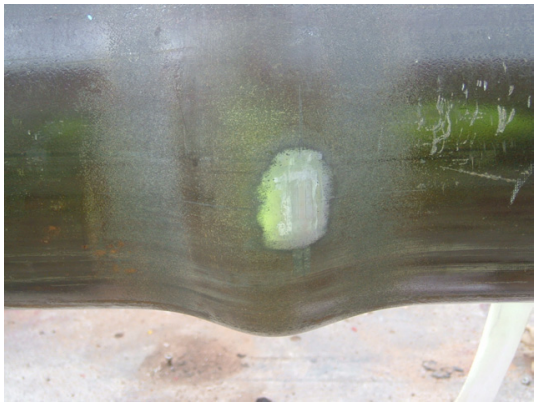

(b)

Figure 8: $\quad$ Plastic deformation in the mild steel pipe caused by the detonation of the PE4 explosive.

In this test, large plastic deformation occurred without any wall failure. The bulging in the pipe is highly localised to the region where the cylindrical charge was placed and cracking in the pipe lacquer (Fig. 8b) in close proximity to the bulging would indicate substantial longitudinal deformation.

\section{Conclusions}

In an attempt to explore the failure process of containment vessels subjected to internal explosive loading, a series of full-scale field tests on steel pipes combined with numerical and analytical modelling was conducted with funding from AWE. The results displayed in this paper are for those pipes that underwent plastic deformation with no wall failure. From the numerical analyses of the 
problem it was found that material behaviour and rate effects are dominant factors that affect the response of the vessel considerably. Hence, it is important to be able to define and model the material properties as accurately as possible in the simulation.

A comparison between the existing and derived theoretical analyses and the numerical modelling showed that the equation proposed by Duffey and Mitchell [1] was a closer approximation to the finite element simulation when determining the final plastic hoop strain in a blast loaded cylindrical shell.

\section{Acknowledgements}

The sponsorship by AWE plc and EPSRC (DTA) has enabled this work to be carried out and is gratefully acknowledged.

\section{References}

[1] Duffey, T. \& Mitchell, D., Containment of explosions in cylindrical shells. International Journal of Mechanical Sciences, 15, pp. 237-249, 1973.

[2] Fanous, F. \& Lowell, G., Simplified analysis for impulsively loaded shells. Journal of Structural Engineering, 114(4), pp. 885-899, 1988.

[3] Clayton, A., Preliminary design of vessels to contain explosions, Proceedings of $11^{\text {th }}$ International Conference on Pressure Vessel Technology, PVP2006-ICPVT11-93735, Vancouver, 2006.

[4] Baker, W., The elastic-plastic response of thin spherical shells to internal blast loading. Journal of Applied Mechanics, 27(1), pp. 139-144, 1960.

[5] ANSYS AUTODYN v.11 (2006) Explicit Software for Non-linear Dynamics. ANSYS, Inc., www.ansys.com.

[6] Johnson, G. \& Cook, W., A constitutive model and data for metals subjected to large strains, high strain rates and high temperatures. Proceedings of the $7^{\text {th }}$ International Symposium on Ballistics, The Hague, pp. 541-547, 1985.

[7] Departments of the US Army, Navy and Air Force. Structures to Resist the Effects of Accidental Explosions, Report TM5-1300, November 1990. 